\title{
Clinical Utility of Arterial Blood Gas Test in an Intensive Care Unit: An Observational Study
}

\author{
Jagadish Chandran ${ }^{1} \odot$, Carol D’Silva ${ }^{2} \odot$, Sampath Sriram ${ }^{3} \odot$, Bhuvana Krishna ${ }^{4} \odot$
}

\begin{abstract}
Background: Arterial blood gas (ABG) analysis is a common test ordered in critically ill patients. Often, it is performed very frequently without influencing patient care. Hence, we decided to check the utility of the ABG test in our intensive care unit (ICU).

Materials and methods: The data of the previous day ABGs were captured by reviewing the chart in an online pro forma which was filled by the authors. Data relating to patient's details, who ordered ABGs, reason for ordering ABGs, and did the ABG influence patient's management were entered. A total of 985 ABGs were performed in 173 patients for 2 months which was analyzed.

Results: Out of 985 ABGs, in 259 instances (26.29\%), interventions were done after reviewing an ABG. The major interventions among these ABGs were ventilator settings adjustment in $134 \mathrm{ABGs}(13.6 \%)$. A total of $790 \mathrm{ABGs}$ were done routinely with no specific indication (80.20\%), while doctors ordered one following an event for 195 ABGs (19.80\%).

Conclusion: Our data suggest that $80 \%$ of $A B G$ tests were ordered as part of a routine test.

Keywords: Arterial blood gas, Arterial cannula, Clinical utility.

Indian Journal of Critical Care Medicine (2021): 10.5005/jp-journals-10071-23719
\end{abstract}

\section{INTRODUCTION}

Arterial blood gas ( $A B G$ ) analysis is one of the most common tests ordered in the ICU. Ideally, an ABG sample should be obtained, when the results are likely to influence patient management. ${ }^{1}$ Common indication for the ABG sample is the need to evaluate the adequacy of patient ventilation, the need to quantify the response to therapeutic or diagnostic interventions, monitoring of severity and progression of a documented disease process, and the assessment of acid-base status. ${ }^{2}$ Repeated ABG measurements are associated with increased costs, blood loss, introduce or spread infection, and patient discomfort. ${ }^{3}$ In patients who do not have an intra-arterial catheter, the need for repeated and uncomfortable punctures can be associated with substantial blood loss. ${ }^{4}$ According to a study in a large academic hospital, only $26.4 \%$ of ABG tests were ordered after an acute respiratory event. ${ }^{1}$ Similarly, other researchers have found that between 42.7 and $66 \%$ of ABG measurements were not clinically justified in their intensive care units (ICUs). ${ }^{5-7}$ In our ICU, $A B G$ tests are performed once in the morning and at fixed intervals, for example, 4th hourly, 6th hourly at the doctor's discretion, irrespective of the patient's condition at that time. Because of the large volume of ABG tests performed in our ICU, we decided to examine the utilization of these routine $A B G s$.

\section{A Im}

To assess the clinical utility of routine ABG tests in our ICU.

\section{Objectives}

Primary: To assess whether the ABG tests are clinically justified. Secondary: To assess the cost-effectiveness of ABGs in the ICU.

\begin{abstract}
${ }^{1-4}$ Department of Critical Care Medicine, St. John's Medical College and Hospital, Bengaluru, Karnataka, India

Corresponding Author: Jagadish Chandran, Department of Critical Care Medicine, St. John's Medical College and Hospital, Bengaluru, Karnataka, India, Phone: +91 9486133459, e-mail: jagge86@gmail.com How to cite this article: Chandran J, D'Silva C, Sriram S, Krishna B. Clinical Utility of Arterial Blood Gas Test in an Intensive Care Unit: An Observational Study. Indian J Crit Care Med 2021;25(2):172-175.
\end{abstract}

Source of support: Nil

Conflict of interest: None

\section{Materials and Methods Data Collection}

This is a prospective observational study conducted in 30 bedded ICU in a tertiary care teaching hospital. This study was approved by the institutional ethical committee with reference number 22/2018, which waived the requirement for informed written consent. Our ICU has adequate manpower with a minimum nursing-to-patient ratio of $1: 2$ and 3 residents per shift covering a maximum of 10 patients per resident and oversee by 3 consultants. The ABG tests are performed in all patients based on ICU protocol at fixed intervals and based on case to case basis at the treating doctor's discretion. All the patients admitted to the medical ICU from June 2018 to July 2018 were included in the study. The data of the previous day $A B G$ s were captured in an online pro forma. It included patient's demographic detail, time of doing ABG, primary diagnosis classified as per ICD 10, comorbid conditions, presence/absence 
of intra-arterial catheter prior, who ordered the $A B G$, reasons for ordering $A B G s$, whether the results were expected, and did the ABG influence patient's management. Interventions were done after an ABG was assessed by reviewing the patient's chart two hours before or after the $A B G$ was performed. Answers were entered in the pro forma by the principal and co-principal investigator. ABGs of patient's age less than 18 years and which were done outside ICU was excluded. All ABGs which were done in the first 24 hours of ICU admission were also excluded in the study because multiple ABG $s$ may be warranted during the initial resuscitation of the patient.

\section{Statistical Analysis}

Results were expressed as median \pm interquartile range for continuous variables and the number (percentage) for dichotomous variables. The association between a number of $A B G$ s and interventions done was analyzed by the chi-square test. A $p$-value of $<0.05$ was considered significant. The analysis was performed using STATA v14 (StataCorp. 2015. Stata Statistical Software: Release 14. College Station, TX: StataCorp LP).

\section{Results}

A total of 985 ABGs were performed in 173 patients for 2 months (June 2018-July 2018). Out of 985 ABGs, in 259 instances (26.29\%), interventions were done after reviewing an ABG. The major interventions among these $A B G s$ were ventilator settings adjustment in $134 \mathrm{ABGs}$ (13.6\%), correction of dyselectrolytemia in $73 \mathrm{ABGs}$ (7.41\%), correction of hypoglycemia in $20 \mathrm{ABGs}(2.03 \%)$, oxygen administration in $8 \mathrm{ABGs}(0.81 \%)$, and other interventions like fluid administration, intubation, extubation, and blood transfusion in $21 \mathrm{ABGs}$ (2.14\%) (Fig. 1).

In a total of $985 \mathrm{ABGs}, 790 \mathrm{ABG}$ s were done based on protocol with no specific indication (80.20\%), while 195 ABGs (19.80\%) were ordered on case to case basis by the treating doctors (Table 1).

The initial primary diagnosis was classified based on the organ system involved using ICD-10 chapters and it was seen that 257 ABGs (26.09\%) were done in patients having a primary disorder of the respiratory system, followed by primary central nervous system (CNS) disease (199, 20.2\%), disease of the digestive system $(138,14.01 \%)$, certain infectious and parasitic diseases $(102,10.36 \%)$,



Fig. 1: Distribution of most common interventions performed following an $A B G$
Table 1: Frequency of ABGs ordered and intervened

\begin{tabular}{llcl}
\hline & \multicolumn{3}{c}{$\begin{array}{c}\text { Any Intervention performed } \\
\text { Numbers (\%) }\end{array}$} \\
\cline { 2 - 3 } Order of $A B G$ & No & \multicolumn{1}{c}{ Yes } & Total \\
\hline Protocol based & $615(77.85)$ & $175(22.15)$ & 790 \\
Case to case basis & $111(56.92)$ & $84(43.08)$ & 195 \\
Total & $726(73.71)$ & $259(26.29)$ & 985 \\
\hline
\end{tabular}

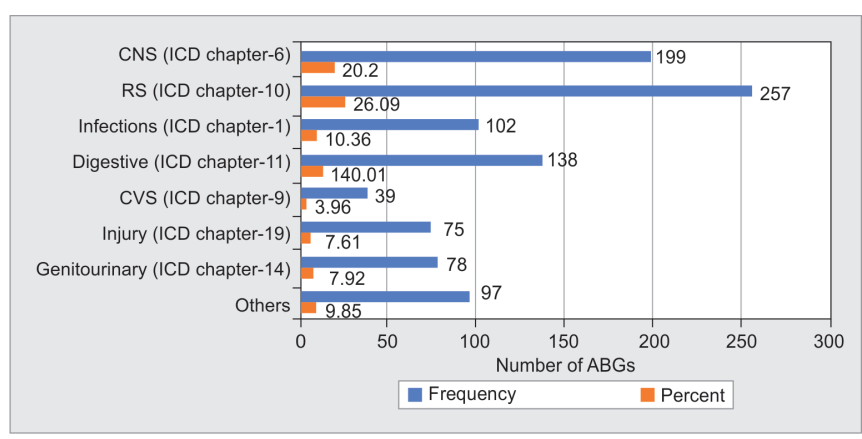

Fig. 2: Number of ABGs performed according to patient diagnosis based on ICD-10 chapters classification

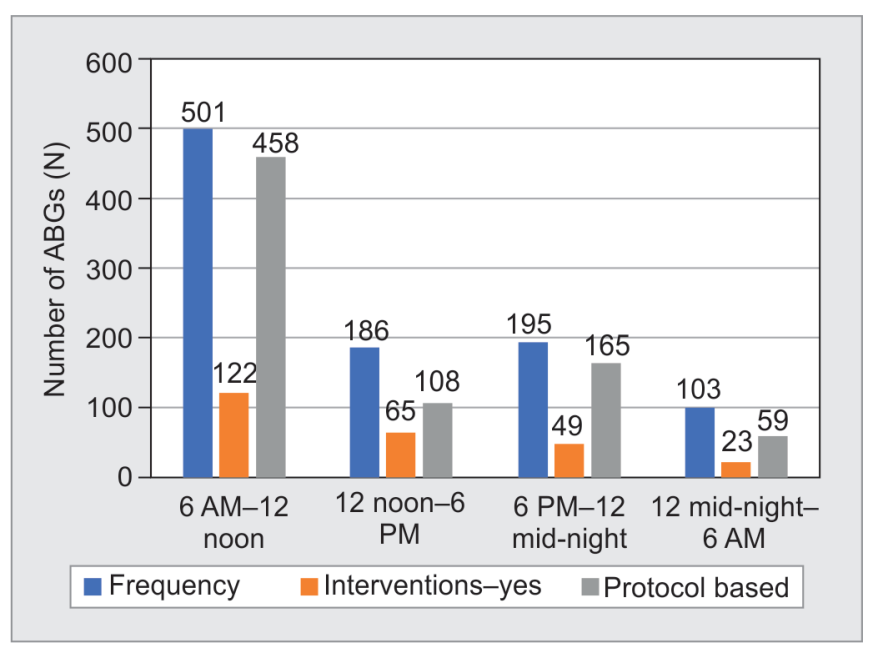

Fig. 3: Time of performing ABGs

injury, poisoning and certain other consequences of an external cause $(75,7.61 \%)$, disease of the genitourinary system $(78,7.92 \%)$, and others (97, 9.85\%) (Fig. 2).

Regarding the time of ABGs performed, 501 ABGs were done between 6 am and $12 \mathrm{pm}$ (50.86\%), 186 ABGs between $12 \mathrm{pm}$ and 6 pm (18.88\%), 195 ABGs between 6 pm and $12 \mathrm{am} \mathrm{(19.8 \% ),} \mathrm{and}$ 103 ABGs between 12 am and 6 am (10.46\%) (Fig. 3).

Patients who were on invasive ventilation had 729 ABGs (74.01\%), patients on supplemental oxygen had 160 ABGs (16.24\%), spontaneously breathing patients had 65 ABGs (6.6\%), and those who were on NIV/BiPAP support had 31 ABGs (3.15\%) (Fig. 4).

Out of 985 ABGs performed, 488 ABGs (49.54\%) were performed in patients who had an invasive arterial cannula (Table 2). 




Fig. 4: Distribution of the types of respiratory support

Table 2: Frequency of $A B G$ s basis the presence of invasive arterial cannula

\begin{tabular}{ll}
\hline Invasive arterial cannula & Frequency (\%) \\
\hline No & $497(50.46)$ \\
Yes & $488(49.54)$ \\
\hline
\end{tabular}

Among the metabolic causes for performing an ABG, such as monitoring/follow-up of electrolytes, glucose, lactate clearance, fluid administration, 921 ABGs (93.5\%) did not have any cause to assess the metabolic status of patients, and 49 ABGs (4.97\%) were performed post dialysis.

\section{Discussion}

The researches in the utilization of $A B G$ tests are limited and have been studied only by a few. The scope of these studies was limited to single parameters like change in ventilator settings, ${ }^{8}$ patient safety, and outcome. ${ }^{9}$ None of the studies discussed the reason for ordering $A B G s$, whether these $A B G$ s were intervened or not.

This two-month observational study conducted on 985 ABGs showed that interventions were performed only in 259 ABGs (i.e., $26.29 \%)$. In that, the major interventions were changed in ventilator settings (13.6\%). Pilon et al. ${ }^{7}$ suggested obtaining ABG tests when there is a change in ventilator settings. The validity of such an indication is questionable in current practice as it is not considered an absolute indication. Additionally, as per Liou et al., ${ }^{10}$ the change of positive end-expiratory pressure was found to have no significant influence on $A B G$ results.

Unlike the study done by Melanson et al. ${ }^{1}$ and MartínezBalzano et al. ${ }^{11}$ on the utilization of ABG tests after an educational or guidelines intervention, our study did not educate or asked the treating doctors to fill the pro forma prior to performing an ABG test. As we want to check the number of $A B G$ tests that were performed and intervened objectively hence to prevent the Hawthorne effect.

Regarding the timing of the $A B G$ test performed, we have grouped the $A B G$ s into four groups with 6 hours intervals. We have observed that the maximum numbers of $A B G$ s were done from $6 \mathrm{AM}$ to 12 noon slot but only $24 \%$ interventions noticed. Maximum interventions were observed from 12 noon to 6 PM slot (35\%) suggesting that the utility of ABG tests was maximum after morning rounds $(p=0.003)$. Most of the studies except Melanson et al. ${ }^{1}$ have not assessed the timing of $A B G$ tests and their significance.

According to Muakkassa et al., ${ }^{12}$ presence of an arterial line was the strongest predictor of the number of $A B G$ tests performed per patient. We discerned no differences as an almost equal number of $A B G s$ were ordered both in the presence and absence of an invasive arterial catheter. We also found a lesser number of interventions were made in both groups.

\section{Limitations}

This was a single-center, observational study. All ABG parameters were not recorded, rather we assessed the outcomes of conducting routine $A B G s$. A total of $73.71 \%$ of $A B G$ s were not followed by an intervention and this may give an impression of $A B G$ sot being useful in those subsets of patients. However, a normal ABG does allow the treating physician to know that patient management is on track. As this study was done objectively, the data collected from ICU charts may be prone to typographical errors.

\section{Conclusion}

Arterial blood gas analysis is often ordered as a routine test and the $A B G$ results do not change patient management in most cases, which results in increased cost and resources. As most of the routine $A B G$ s go non- intervened, an individualized approach may reduce the number of inappropriate ABGs.

\section{ORCID}

Jagadish Chandran (ㄱ) https://orcid.org/0000-0001-9651-6789

Carol D'Silva @ittps://orcid.org/0000-0002-3920-1366

Sampath Sriram @ https://orcid.org/0000-0001-7521-7106

Bhuvana Krishna @i https://orcid.org/0000-0002-0003-6797

\section{References}

1. Melanson SE, Szymanski T, Rogers SO, Jarolim P, Frendl G, Rawn JD, et al. Utilization of arterial blood gas measurements in a large tertiary care hospital. Am J Clin Pathol 2007;127(4):604-609. DOI: 10.1309/ ELH5BPQ0T17RRKOM.

2. AARC clinical practice guideline. Sampling for arterial blood gas analysis. American Association for Respiratory Care. Respir Care 1992;37(8):913-917.

3. Andrews $\mathrm{T}$, Waterman $\mathrm{H}$, Hillier V. Blood gas analysis: a study of blood loss in intensive care. J Adv Nurs 1999;30(4):851-857. DOI: 10.1046/j.1365-2648.1999.01155.x.

4. Smoller BR, Kruskall MS. Phlebotomy for diagnostic laboratory tests in adults. Pattern of use and effect on transfusion requirements. N Engl J Med 1986;314(19):1233-1235. DOI: 10.1056/ NEJM198605083141906.

5. Merlani P, Garnerin P, Diby M, Ferring M, Ricou B. Quality improvement report: linking guideline to regular feedback to increase appropriate requests for clinical tests: blood gas analysis in intensive care [published correction appears in BMJ 2001 Oct 27;323(7319):993]. BMJ 2001;323(7313):620-624. DOI: 10.1136/ bmj.323.7313.620.

6. Browning JA, Kaiser DL, Durbin CG. The effect of guidelines on the appropriate use of arterial blood gas analysis in the intensive care unit. Respir Care 1989;34(4):269-276. 
7. Pilon CS, Leathley M, London R, McLean S, Phang PT, Priestley R, et al. Practice guideline for arterial blood gas measurement in the intensive care unit decreases numbers and increases appropriateness of tests. Crit Care Med 1997;25(8):1308-1313. DOI: 10.1097/00003246$199708000-00016$.

8. Al Ashry HS, Richards JB, Fisher DF, Sankoff J, Seigel TA, Angotti LB, et al. Emergency department blood gas utilization and changes in ventilator settings. Respir Care 2018;63(1):36-42. DOI: 10.4187/respcare.05590.

9. Blum FE, Lund ET, Hall HA, Tachauer AD, Chedrawy EG, Zilberstein J. Reevaluation of the utilization of arterial blood gas analysis in the Intensive Care Unit: effects on patient safety and patient outcome. J Crit Care 2015;30(2):438.e1-438.e5. DOI: 10.1016/j.jcrc.2014.10.025.
10. Liou CM, Lin CH, Kang HM, Liu YC, Tso HS. The influence of position and PEEP on arterial blood gas during operation. Ma Zui Xue Za Zhi 1993;31(2):103-112.

11. Martínez-Balzano CD, Oliveira P, O'Rourke M, Hills L, Sosa AF; Critical Care Operations Committee of the UMass Memorial Healthcare Center. An educational intervention optimizes the use of arterial blood gas determinations across ICUs from different specialties: a qualityimprovement study. Chest 2017;151(3):579-585. DOI: 10.1016/j. chest.2016.10.035.

12. Muakkassa FF, Rutledge R, Fakhry SM, Meyer AA, Sheldon GF. ABGs and arterial lines: the relationship to unnecessarily drawn arterial blood gas samples. J Trauma 1990;30(9):1087-1095. 